\title{
Phototheranostic Porphyrin Nanoparticles Enable Vis- ualization and Targeted Treatment of Head and Neck Cancer in Clinically Relevant Models
}

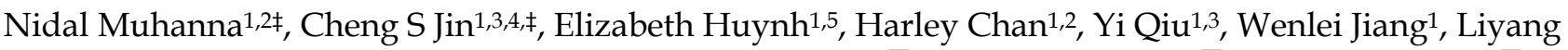 \\ Cui $^{1,5,6}$, Laura Burgess ${ }^{1,5}$, Margarete K Akens ${ }^{1,5}$, Juan Chen ${ }^{\bowtie}$, Jonathan C Irish ${ }^{1,2}{ }^{\bowtie}$, Gang Zheng ${ }^{1,3,4,5}$ \\ 1. Princess Margaret Cancer Centre and Techna Institute, University Health Network, Toronto, Canada. \\ 2. Department of Otolaryngology-Head and Neck Surgery, University of Toronto, Toronto, Canada \\ 3. Department of Pharmaceutical Sciences, University of Toronto, Toronto, Canada \\ 4. Institute of Biomaterials and Biomedical Engineering, University of Toronto, Toronto, \\ 5. Department of Medical Biophysics, University of Toronto, Toronto, Canada \\ 6. Medical Isotopes Research Center, Peking University, Beijing, China \\ ‡ First two authors contributed equally to this work.
}

$\triangle$ Corresponding authors: Dr. Gang Zheng, University of Toronto, TMDT 5-354, 101 College Street, Toronto, ON, M5G 1L7, Canada, E-mail: gang.zheng@uhnres.utoronto.ca. Dr. Jonathan C Irish, University of Toronto, 190 Elizabeth Street, Toronto, Ontario, M5G 2C4, Canada, E-mail: Jonathan.Irish@uhn.ca. Dr. Juan Chen, UHN, TMDT 5-353, 101 College Street, Toronto, ON, M5G 1L7, Canada, E-mail: juan.chen@uhnresearch.ca

(c) 2015 Ivyspring International Publisher. Reproduction is permitted for personal, noncommercial use, provided that the article is in whole, unmodified, and properly cited. See http://ivyspring.com/terms for terms and conditions.

Received: 2015.08.04; Accepted: 2015.08.26; Published: 2015.10.18

\begin{abstract}
Head and neck cancer is the fifth most common type of cancer worldwide and remains challenging for effective treatment due to the proximity to critical anatomical structures in the head and neck region, which increases the probability of toxicity from surgery and radiotherapy, and therefore emphasizes the importance of maximizing the targeted ablation. We have assessed the effectiveness of porphysome nanoparticles to enhance fluorescence and photoacoustic imaging of head and neck tumors in rabbit and hamster models. In addition, we evaluated the effectiveness of this agent for localized photothermal ablative therapy of head and neck tumors. We have demonstrated that porphysomes not only enabled fluorescence and photoacoustic imaging of buccal and tongue carcinomas, but also allowed for complete targeted ablation of these tumors. The supremacy of porphysome-enabled photothermal therapy over surgery to completely eradicate primary tumors and metastatic regional lymph node while sparing the adjacent critical structures' function has been demonstrated for the first time. This study represents a novel breakthrough that has the potential to revolutionize our approach to tumor diagnosis and treatment in head and neck cancer and beyond.
\end{abstract}

Key words: Porphyrin, Nanoparticle, Fluorescence, Photoacoustic imaging, Photothermal therapy

\section{Introduction}

Head and neck squamous cell carcinomas (HNSCC) frequently metastasize to the lymph nodes of the neck, which contributes to a low five-year survival rate of $66 \%$ [1]. Management of HNSCC depends on the anatomical site of the primary tumor, the stage of disease, as well as patient comorbidities. While surgical resection is the preferred treatment for oral cavity (floor of mouth, hard palate, buccal, retromolar trigone, tongue) and sinonasal cancers, radiation-based regimens with chemotherapy, focusing on organ preservation are employed for oropharynx, nasopharynx, hypopharynx and laryngeal carcinomas $[2,3]$. Currently, both surgery and radiation with chemotherapy have significant side effects with acute toxicities and long-term morbidities [4-6]. Therefore, developing advanced imaging modalities 
for the detection of primary and metastatic disease combined with novel targeted ablative therapeutics with high efficacy, high selectivity, and minimal toxicity are crucial.

In recent years, photothermal therapy (PTT) has emerged as a widely investigated tool for cancer treatment [7]. This technique requires PTT agents to convert light at certain wavelengths into heat to destroy surrounding tumor cells through thermally induced necrosis. Thus, PTT has the potential to improve the specificity of HNSCC treatment by localizing laser irradiation and enhancing tumor-accumulation of PTT agents, thus minimizing comorbidities to surrounding healthy tissues. Furthermore, PTT agents are capable for photoacoustic imaging due to their heat generation properties upon irradiation and may potentially serve as an alternative to conventional therapy because of its multifunctionality and photothermal capabilities [8,9].

We discovered a novel multifunctional nanoparticle, porphysome, which consists of porphyrin-lipid building blocks that self-assemble into liposome-like nanoparticles (100-150 nm diameter) [10]. Prior to self-assembly, porphyrin-lipids are highly fluorescent, but upon self-assembly the highly dense packing of porphyrin-lipids makes the porphysome highly absorptive but fluorescence-silent, which results in efficient conversion of light energy to heat upon laser irradiation to provide ideal photothermal and photoacoustic properties that are unprecedented for organic nanoparticles [10]. Porphysomes have been demonstrated to be sensitive photoacoustic imaging agents for sentinel lymph node mapping and as potent PTT agents for effective ablation tumor under both hyperoxic and hypoxic conditions [10, 11]. The nano-scaled size of the porphysome allows for preferential accumulation in primary tumors and metastatic tumor via the enhanced permeability and retention (EPR) effect [10, 12]. Porphysomes dissemble gradually upon the accumulation at interstitial space of tumor. The fluorescence of disrupted porphysomes is unquenched which facilitates the delineation of tumors, while the remaining intact porphysomes enable tumor-specific PTT. Moreover, because of the natural chlorophyll origin of porphyrin, porphysomes are biodegradable and have very low toxicity in vivo $(1000 \mathrm{mg} / \mathrm{kg}$ intravenous injection in mice with no significant negative functional, hematological or histological effects) $[10,11]$. Considering their biocompatibility and robust fabrication, porphysomes have great potential for clinical and commercial translation compared to other inorganic PTT agents, such as graphene [13], gold nanoshells [14, 15], nanorods [16, 17], and nanocages [18]. Taken together, porphysomes might serve as potential alternatives to the conventional HNSCC therapy for enhancing treatment specificity. The tumor surgical resection and localized PTT could be guided by both fluorescence and photoacoustic imaging to enhance treatment specificity, while preserving the anatomical and functional integrity vital structures in the head and neck area.

Here, we present pre-clinical evidence that demonstrates successful fluorescence and photoacoustic image-guided detection of HNSCCs in both a buccal mucosa squamous cell carcinoma rabbit model and a hamster cheek carcinogenesis model. In addition, we demonstrate for the first time, the advantages of porphysome-enabled PTT compared to conventional surgical resection of HNSCC to completely eradicate the tumor(s) and lymph node metastasis while maintaining the integrity of vital anatomical structures (Figure 1). Therefore, this single porphyrin nanotheranostic agent that combines photoacoustic and fluorescence imaging to guide HNSCC surgeries and intervention, along with localized photothermal treatment could be a great potential to improve the specificity and therapeutic efficacy of HNSCC treatment.

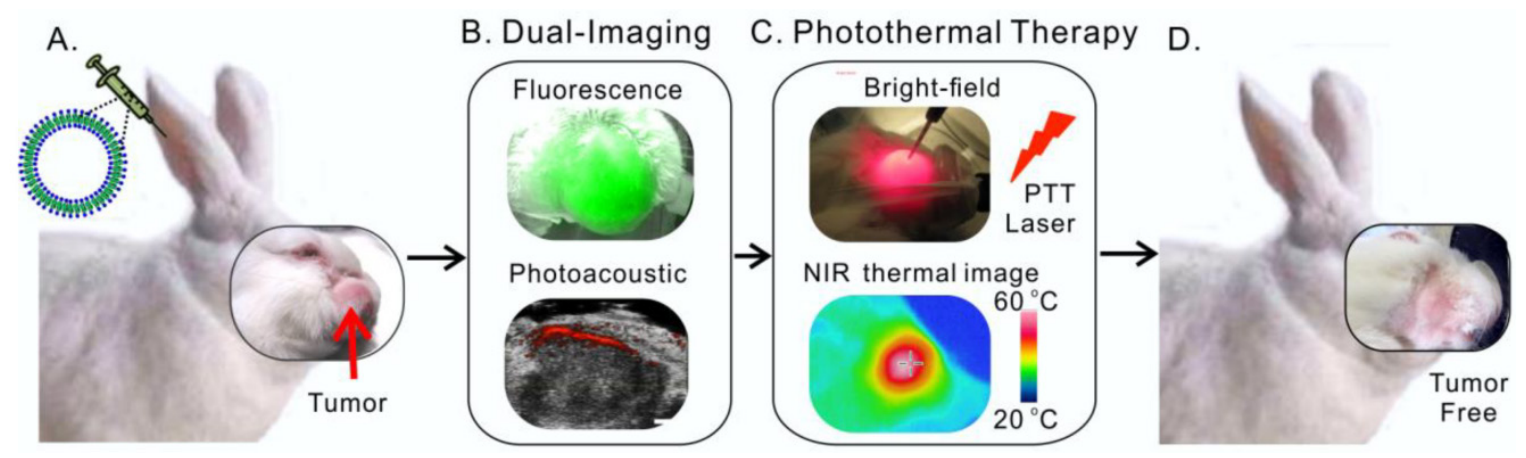

Figure 1. Scheme of multifunctional porphysome-enabled visualization and photothermal therapy on orthotopic rabbit head and neck cancer. A, porphysomes were injected intravenously via ear vein of the rabbit; B, at $24 \mathrm{~h}$ post-injection, both fluorescence and photoacoustic imaging were enabled by porphysomes accumulated at rabbit tumor; $\mathbf{C}$, in vivo photothermal ablation of rabbit tumor by two-step ablations, including one intra-tumor and one transdermal irradiation; $\mathbf{D}$, the orthotopic head and neck tumor was completely eliminated with no recurrence. 


\section{Results}

\section{Tracking porphysome status by dual imaging modalities}

In essence, photoacoustic and fluorescence imaging are two individual imaging properties of porphysomes, which are characteristic of distinct structural forms of porphysomes. While photoacoustic imaging is a nanostructure-driven property of intact porphysomes, fluorescence imaging is generated by free porphyrins upon porphysome nanostructure dissociation [10]. In order to assess the structural status of porphysome (size 117.8 $\pm 2.0 \mathrm{~nm}$, Figure S1) in the blood circulation in vivo, we first examined the photoacoustic and fluorescence imaging capability of the porphysome in bovine serum $(90 \%$ volume fetal bovine serum in PBS). As shown in Figure 2A, porphysomes with highly dense packing of porphyrins did not exhibit significant fluorescence at the beginning of mixing with serum under Cri Maestro ${ }^{\mathrm{TM}}$ imager, but displayed significant photoacoustic signal upon laser irradiation as the result of efficient conversion of light energy to heat, leading to thermoelastic expansion and acoustic waves. After $6 \mathrm{~h}$ incubation, the samples showed progressive increase of fluorescence, while displayed constantly stable photoacoustic signal within $48 \mathrm{~h}$. The fluorescence genera- tion of porphysome in serum was also monitored by Fluoromax ${ }^{\circledR}-4$ fluorescence spectrophotometer, which displayed the same trend of gradual increase with time, further reaffirming the findings observed by Maestro ${ }^{\mathrm{TM}}$ imager (Figure 2B). After $48 \mathrm{~h}$ incubation with serum, the samples' fluorescence signal increased remarkably by 12.6 -fold, but remained still highly-quenched $(90 \%$ fluorescence-quenching efficiency) when compared to that of the completely dissociated sample in 0.5\% Triton X-100 (Figure 2C). These observations suggest that: 1) porphysome nanostructures are quiet stable in the serum; 2) at $48 \mathrm{~h}$ post-incubation, a small fraction of dissociated porphysomes was sufficient to generate a strong fluorescence signal to enable fluorescence imaging; 3) most porphysomes remain intact for efficient conversion of light energy to heat upon laser irradiation, thus enabling photoacoustic imaging and photothermal therapy. Therefore, these dual-imaging modalities might provide complementary information to assess porphysome status after delivery in order to guide treatment planning. The fluorescence imaging could serve as a sensitive tool to track porphysome delivery, while photoacoustic signal could potentially report the amount of intact nanoparticles to guide PTT dosimetry.

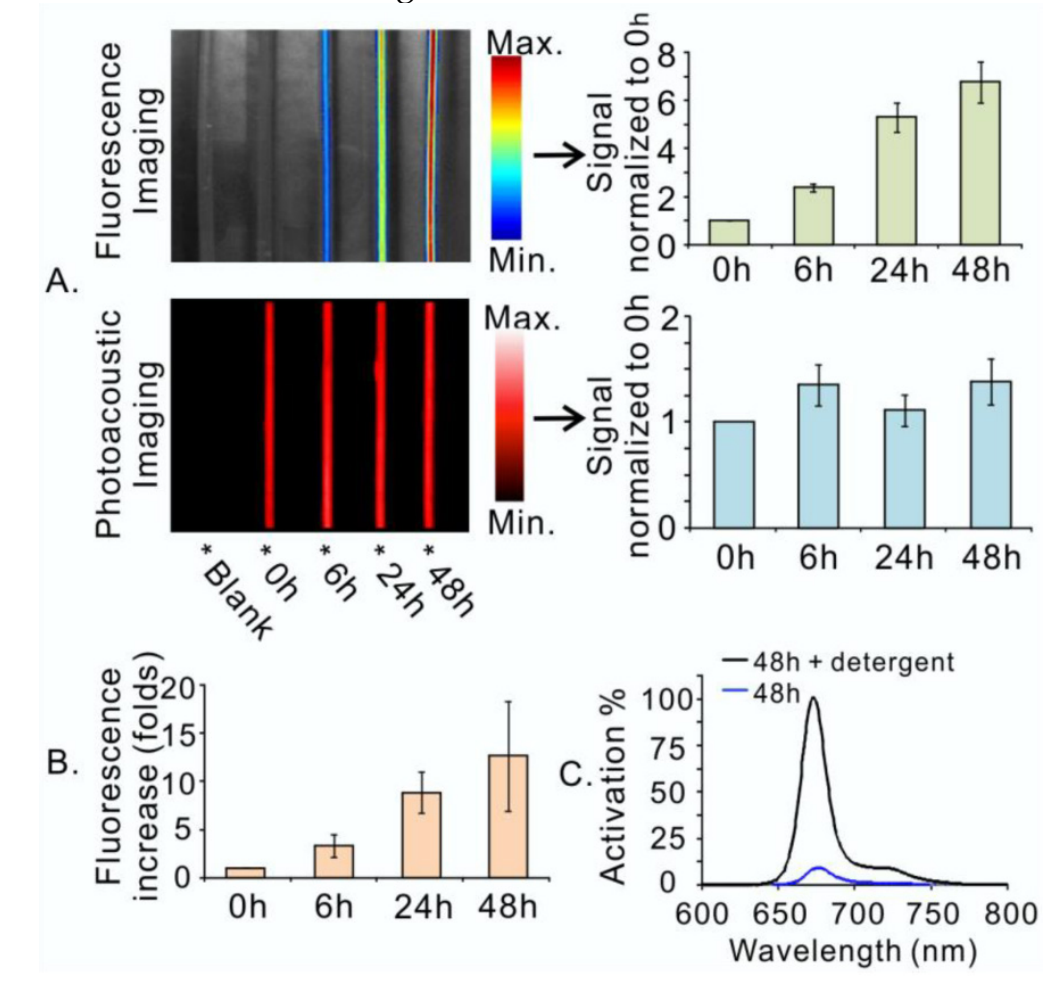

Figure 2. Porphysomes enabled dual-imaging (fluorescence and photoacoustic). A, Porphysome stability in serum was assessed by dual-imaging (left), and their signals were quantified and normalized to their corresponding image at $0 \mathrm{~h}$ (right); $\mathbf{B}$, The fluorescence increase of porphysome in serum was also quantified with the time by fluorescence spectrophotometer and represented as the increase-fold (normalized to the samples' fluorescence at $0 \mathrm{~h}$ ); $\mathbf{C}$, The percentage of fluorescence generation of porphysome sample at $48 \mathrm{~h}$ post serum incubation was measured by fluorescence spectrophotometer and normalized to the florescence of the completely dissociated sample in $0.5 \%$ Triton $\mathrm{X}-100$. 


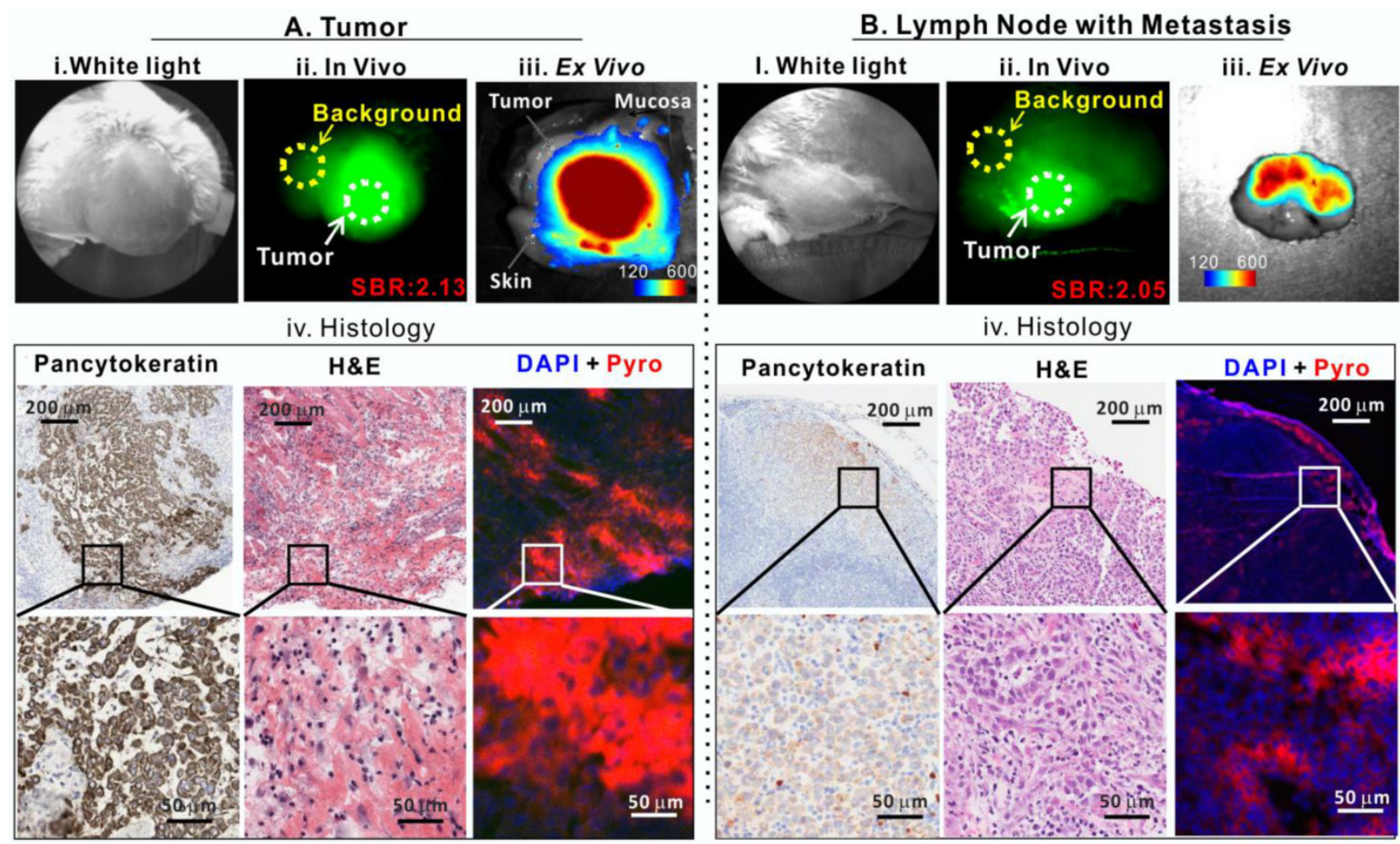

Figure 3. Porphysomes enabled fluorescence delineation of buccal tumor and lymph node metastasis in rabbit. A, Tumor images: i) bright field white-light image, ii) in vivo fluorescence image of tumor with signal-to-background ratio (SBR) of 2.13, iii) ex vivo fluorescence image of tumor (scale bar: 120-600), and iv) histological analysis of frozen tumor tissue by pancytokeratin staining, H\&E staining and fluorescence microscopy; B, metastatic lymph node images: i) bright field white-light image, ii) In vivo fluorescence image of lymph node with metastasis with SBR of 2.05, iii) ex vivo fluorescence image of lymph node with metastasis (scale bar: $120-600$ ), and iv) histological analysis of frozen lymph node slides by pancytokeratin stain, H\&E and fluorescence microscopy.

\section{Porphysome-fluorescence enables delineation of buccal carcinoma in rabbit model}

Our previous study demonstrated that porphysomes enabled clear delineation of orthotopic prostate tumor from normal prostate tissues by fluorescence imaging $24 \mathrm{~h}$ after intravenous injection [12]. To explore the clinical potential of porphysomes for HNSCC management, we first investigated their behavior on a VX2 oral squamous cell carcinoma rabbit model. With the intrinsic copper-64 labeling, we were able to non-invasively track porphysomes and observed their high accumulation in tumor tissue (19.3 \pm $6.8 \% \mathrm{ID} / \mathrm{g})$ and metastatic lymph node (4.9 \pm $0.1 \% \mathrm{ID} / \mathrm{g}$ ) of the rabbit model. We investigated the fluorescence imaging capability of porphysomes for VX2 tumor detection. No significant fluorescence signal was observed in vivo immediately following the intravenous porphysome injection (data not shown). At $24 \mathrm{~h}$ post-injection, the tumor exhibited strong porphyrin fluorescence, with an average tumor signal to background ratio (SBR, tumor to surrounding muscle ratio) of $2.13 \pm 0.49(\mathrm{n}=5)$ (Figure 3A), which indicates that porphysomes preferentially accumulated in the tumor and gradually disassociated to release significantly the fluorescence for tumor visuali- zation. Physical examination of the rabbit by palpation revealed an enlarged cervical lymphadenopathy (Figure 3B, i) that exhibited in vivo fluorescence with an average SBR of $2.05 \pm 0.11$ folds $(n=5)$ (Figure 3B, ii). Consecutively, the tumor (with skin and mucosa intact) and lymph node were dissected with the fluorescence guidance and subjected to ex vivo fluorescence imaging. The tumor showed significantly higher fluorescence than surrounding skin and mucosa (Figure 3A, iii). Similarly, the suspected metastatic lymph node displayed strong fluorescence, indicating a higher accumulation of porphysomes (Figure 3B, iii). The fluorescent tumor and lymph node were subjected to histopathological analyses that confirmed their malignant phenotype as H\&E staining showed cancer cell morphology (e.g. enlarged nuclei, prominent nucleoli and irregular shape) and positive pancytokeratin (PanCK) staining implied the presence of epithelial cells (Figure 3A-3B, iv). In addition, microscopy imaging revealed that cells exhibiting porphysome fluorescence corresponded to tumor cells identified by histopathological analysis in both the tumor (Figure 3A, iv) and lymph nodes (Figure 3B, iv), indicating tumor-specific accumulation of porphysomes at the cellular level. Thus, it appears that porphysomes specifically accumulate in both 
primary tumors and metastatic lymph nodes while sparing surrounding healthy tissues, thus enabling clearly delineation of these malignant tissues to guide surgical resection.

\section{Porphysomes enable photoacoustic imaging of buccal tumor in rabbit model}

As demonstrated in the serum study, a small portion of porphysomes dissociation releases significantly porphyrin fluorescence, while the main portion of intact porphysomes allows for stable photoacoustic imaging (Figure 2A). After demonstrating that porphysome preferentially accumulated in tumor and enabled fluorescence delineation of tumors at $24 \mathrm{~h}$ post administration, we next investigated whether porphysomes also enabled delineation of tumor boundaries by trans-oral photoacoustic imaging in this HNSCC rabbit model. At $24 \mathrm{~h}$ post intravenous injection of porphysomes, the healthy cheek side showed minimal photoacoustic signal whereas the tumor boundary was clearly visible with a 4-fold higher photoacoustic signal compared to the healthy tissue (Figure 4A). Interestingly, topical subcutaneous injection of porphysomes surrounding the tumor area (similar to sentinel lymph node biopsy (SLNB) procedures using technetium-99) enabled the identification of malignant sentinel lymph node (Figure 4B, i, LN2\#) with a higher photoacoustic signal compared to the healthy lymph node (Figure 4B, i, LN\#1). Their corresponding tissue fluorescence microscopy and histopathological analysis (Figure 4B, ii \&iii) reaffirmed the specific accumulation of porphysomes in malignant lymph node, but not in the healthy lymph node. Therefore porphysome-enabled photoacoustic imaging could potentially serve as a diagnostic tool to identify tumors and malignant lymph nodes, and further to report the amount of intact nanoparticles to guide PTT.

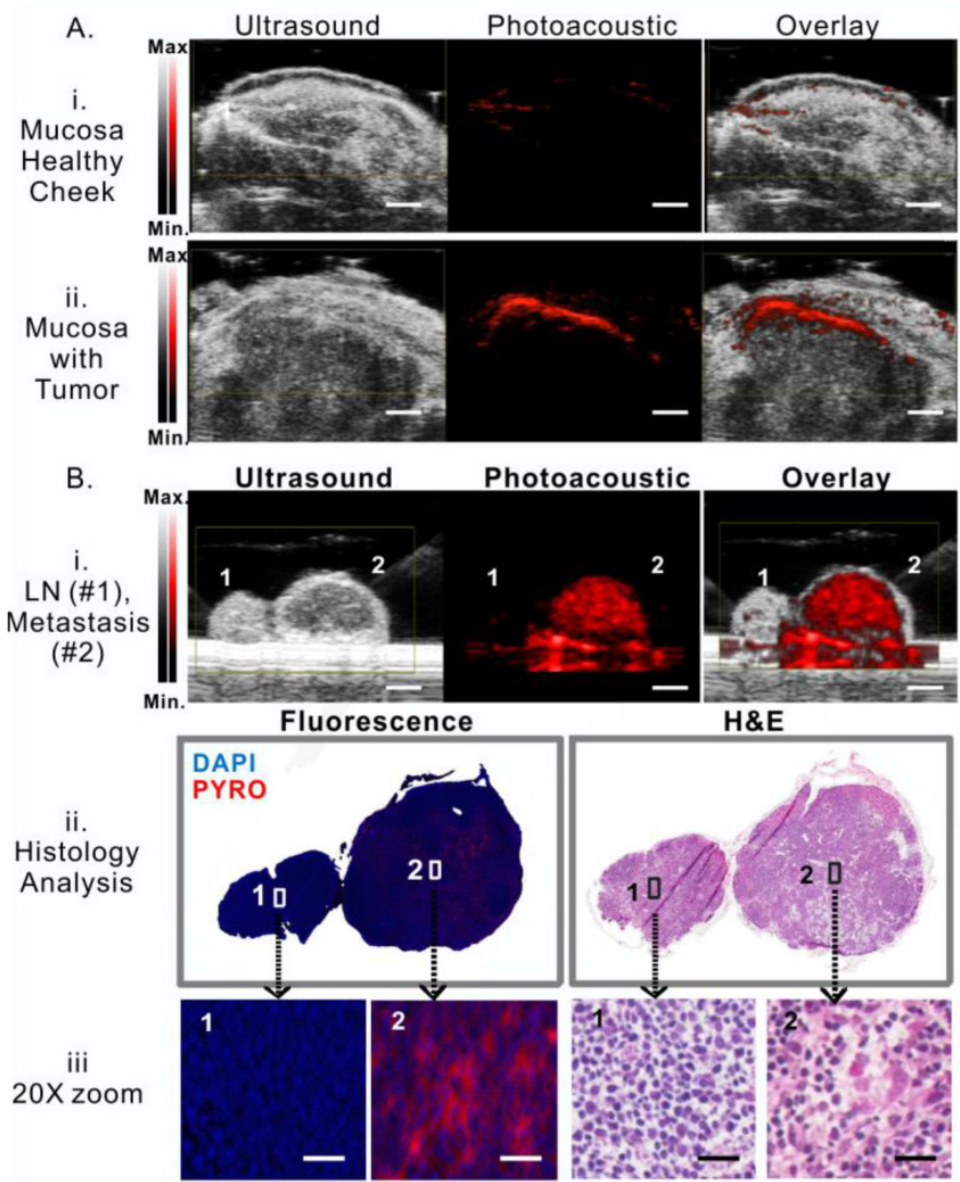

Figure 4. Porphysome-enabled photoacoustic imaging of buccal tumor and lymph node metastasis in rabbit model. A, detection of tumor underneath mucosa by photoacoustic imaging at 24 h post-i.v. injection of porphysome, comparing the healthy cheek (i) and the cheek with tumor (ii) from the same rabbit (scale bar: 3 mm); B, (i) detection of metastasis in lymph nodes by photoacoustic imaging following the intra-tumor injection of porphysomes (scale bar: 3 mm), (ii) Identification of malignancy of lymph node by histological analysis (H\&E staining ) and fluorescence microscopy, (iii) zoom-in image with morphological details showing the accumulation of porphysomes in the metastatic tumor cells in lymph nodes (scale bar: $50 \mu \mathrm{m}$ ). 

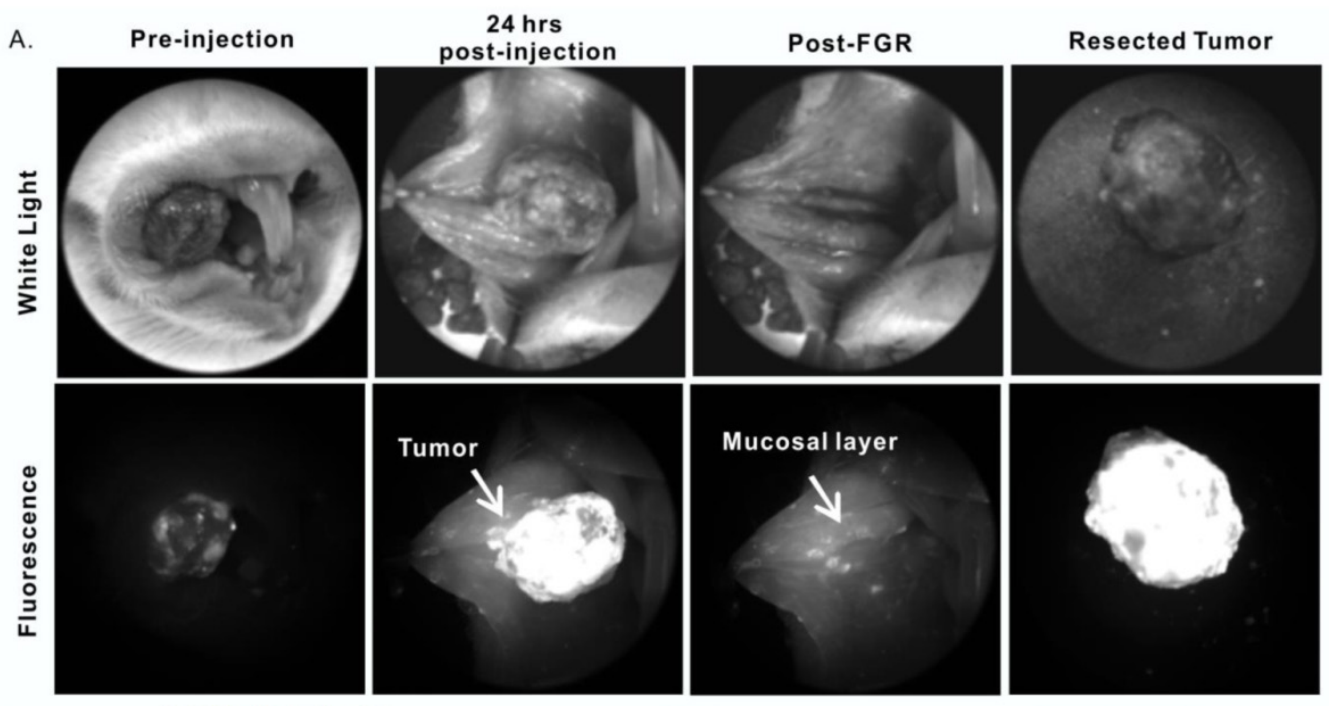

B.

DAPI + Porphyrin

H\&E

Pancytokeratin
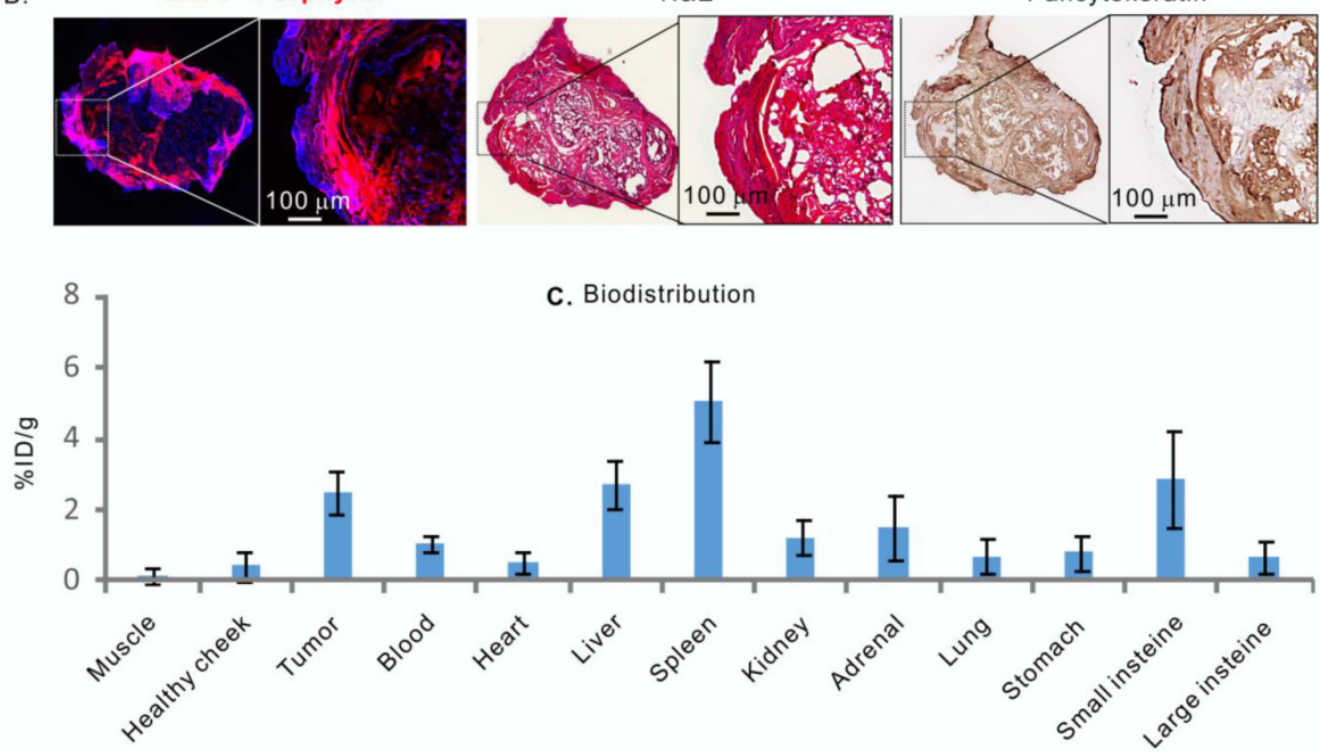

c. Biodistribution

Figure 5. Porphysomes' behaviour in a hamster oral carcinogenesis model. A, in vivo fluorescence imaging of hamster oral tumor and fluorescence-guided resection (FGR); B, Histological analysis of ressected tumor by fluorescence microscopy, H\&E and pancytokeratin stain; C, biodistribution of porphysome in hamster, shown as $\% / D / g, n=3$ to calculate mean and standard deviation.

\section{Porphysomes for HNSCC management in a carcinogenesis model}

Following the confirmation of dual-imaging proficiency of porphysomes in a large orthotopic rabbit model, we then evaluated the theranostic abilities of porphysomes in a more biologically relevant hamster cheek carcinogenesis, which elicited by the repeated treatment of DMBA [19], and closely mimics the clinical manifestations of oral carcinoma in humans [20]. As shown in Figure 5A, tumor-specific strong fluorescence was detected at $24 \mathrm{~h}$ post-injection of porphysomes, providing guidance for tumor resection. All the fluorescent tissues were removed under real-time fluorescence-guided resection until no significant fluorescence was observed at the surgical bed. The animals were then sacrificed, and the dissected tissues and the cheek tissue at the surgical bed were sent for histopathological analysis. Interestingly, the resected tumor under fluorescence guidance showed clear capsulation of the tumor tissue, confirming the complete resection based on the oncological principles under image guidance (Figure 5). The tumor cells located on the dissected fluorescent mucosa were demonstrated by H\&E staining and high positive PanCK staining (Figure 5B), while the surgical bed with negligible fluorescence showed no sign of tumor cell. The tumor area identified by PanCK and H\&E staining were co-localized well with the porphyrin-fluorescent area under microscopy, confirming the tumor-specific accumulation of porphysomes (Figure 5B), and this was further demonstrated by the biodistribution analysis that showed an average accumula- 
tion of $3.85 \pm 0.97 \% \mathrm{ID} / \mathrm{g}$ in tumor versus $0.29 \pm 0.15 \%$ ID/g in healthy oral mucosa $(n=3)$ (Figure 5C). Collectively, the in vivo fluorescence, fluoro-histopathology, and biodistribution analyses demonstrate the tumor-specific accumulation of porphysomes in carcinogenesis hamster model.

Porphysome-enabled photoacoustic imaging was also examined in the hamster oral carcinoma model. Prior to porphysome injection, in vivo photoacoustic imaging of the tumors did not show an observable photoacoustic signal (Figure 6A, i), while at $24 \mathrm{~h}$ post-injection, the tumor boundaries were clearly defined by a prominent photoacoustic signal that was approximately five-fold greater than the pre-injection signal (Figure 6A, ii). The photoacoustic signal of tumors indicated the existence of intact porphysomes and could potentially report the amount of intact na- noparticles.

Guided with porphysome-fluorescence imaging at $24 \mathrm{~h}$ post-injection, a PTT laser was applied for tumor-localized treatment at wavelength of $671 \mathrm{~nm}$. The tumor temperature increased from $30.94{ }^{\circ} \mathrm{C}$ to $62.33{ }^{\circ} \mathrm{C}$ during 100 seconds of irradiation (Figure 6B, $\mathbf{n}=3$ ), indicating that tumor-specific accumulation of porphysomes significantly enhanced heat generation under laser irradiation. Subsequently, the PTT treatment efficacy was then evaluated by histology analysis, which showed that the tumor from the control group with only porphysomes injection (no laser treatment) did not exhibit signs of apoptosis or necrosis, while the tumors that received porphysome-PTT showed significant signs of necrosis and cellular damage, which is indicative of acute thermal damage (Figure 6C).
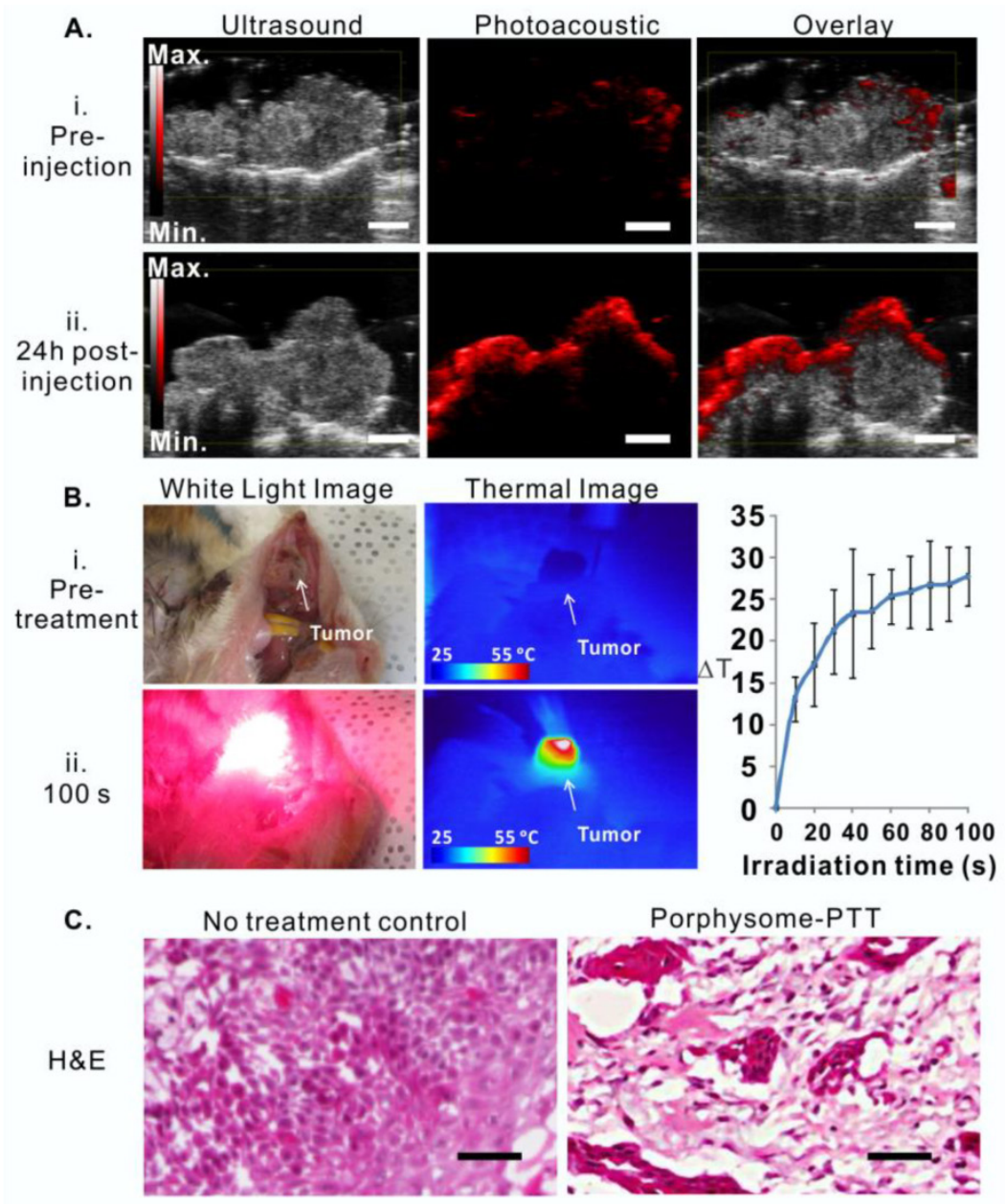

Figure 6. Porphysome-enabled photoacoustic imaging-guided PTT in treating hamster oral carcinoma. A, Detection of hamster oral tumor by photoacoustic imaging at $24 \mathrm{~h}$ post-injection of porphysomes (scale bar: $3 \mathrm{~mm}$ ); B, bright field image (i) and thermal image (ii) of tumor before and 100s post laser irradiation. Temperature increase during PTT irradiation were monitored by thermal camera, $n=3$ for each group to calculate mean and standard deviation; $\mathbf{C}$, Thermal efficacy was evaluated by H\&E histological analysis of tumor tissue with no treatment and porphysome-PTT group (scale bar: $100 \mu \mathrm{m}$ ). 
A. Twp-step PTT

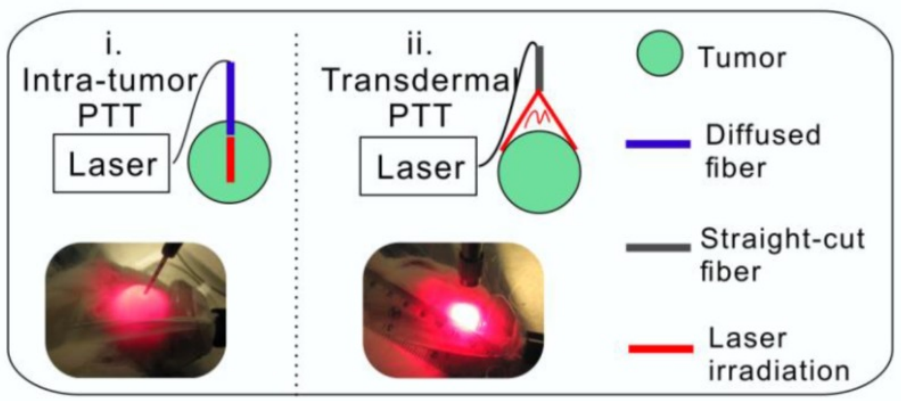

C. Porphysome-PTT In Vivo Images

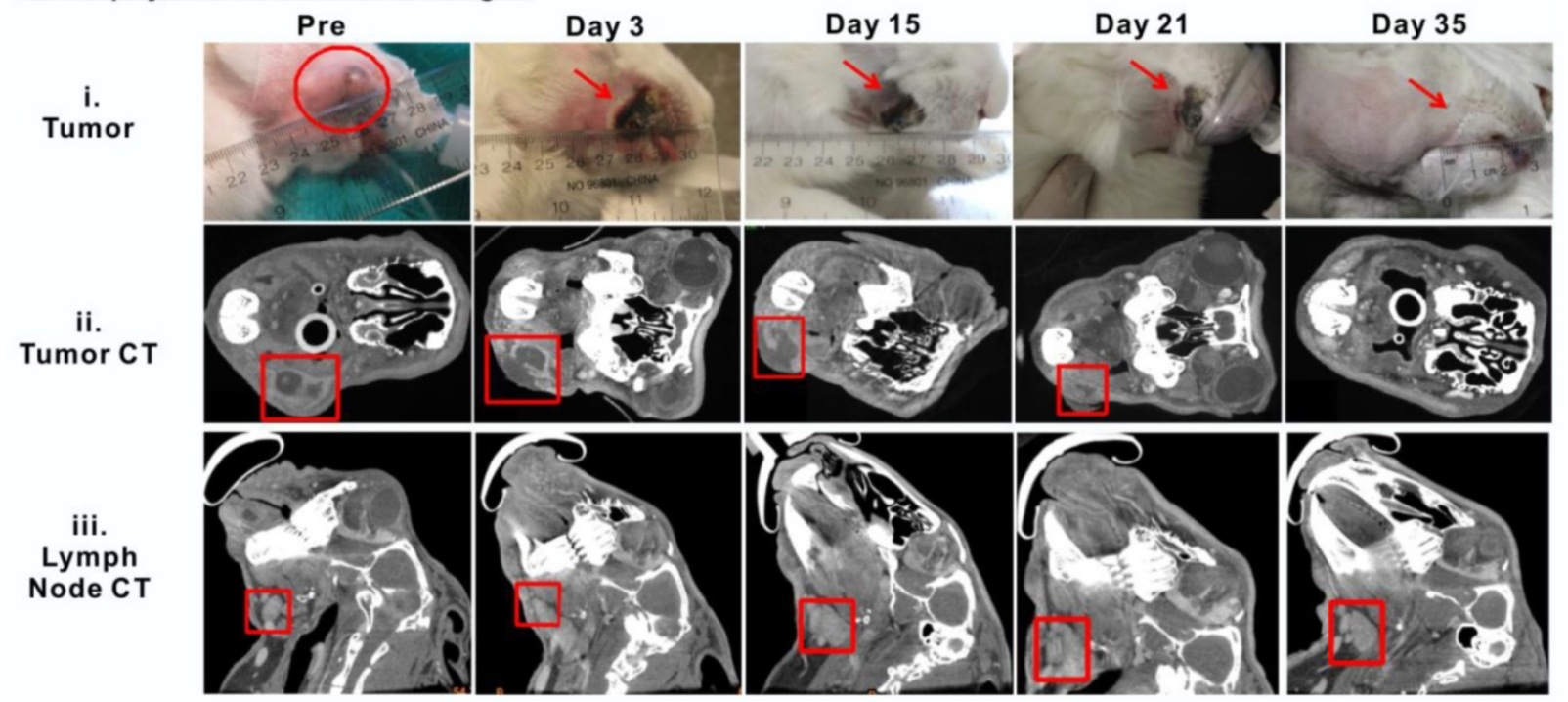

D. Histology Analysis of Rabbit Cheek on Day 35 post-treatment

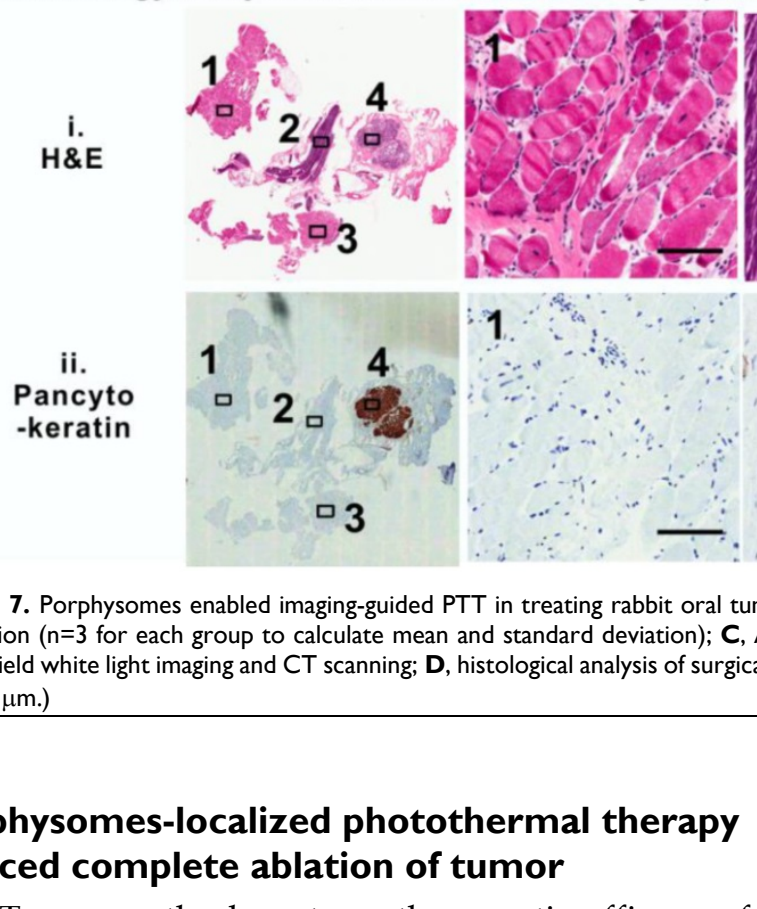

To assess the long-term therapeutic efficacy of PTT on the buccal carcinoma rabbit model, each tumor received two rounds of PTT irradiation in order to cover the entire tumor area due to the large tumor size (Figure 7A). The first PTT treatment was con-

\section{B. Tumor Temperature}
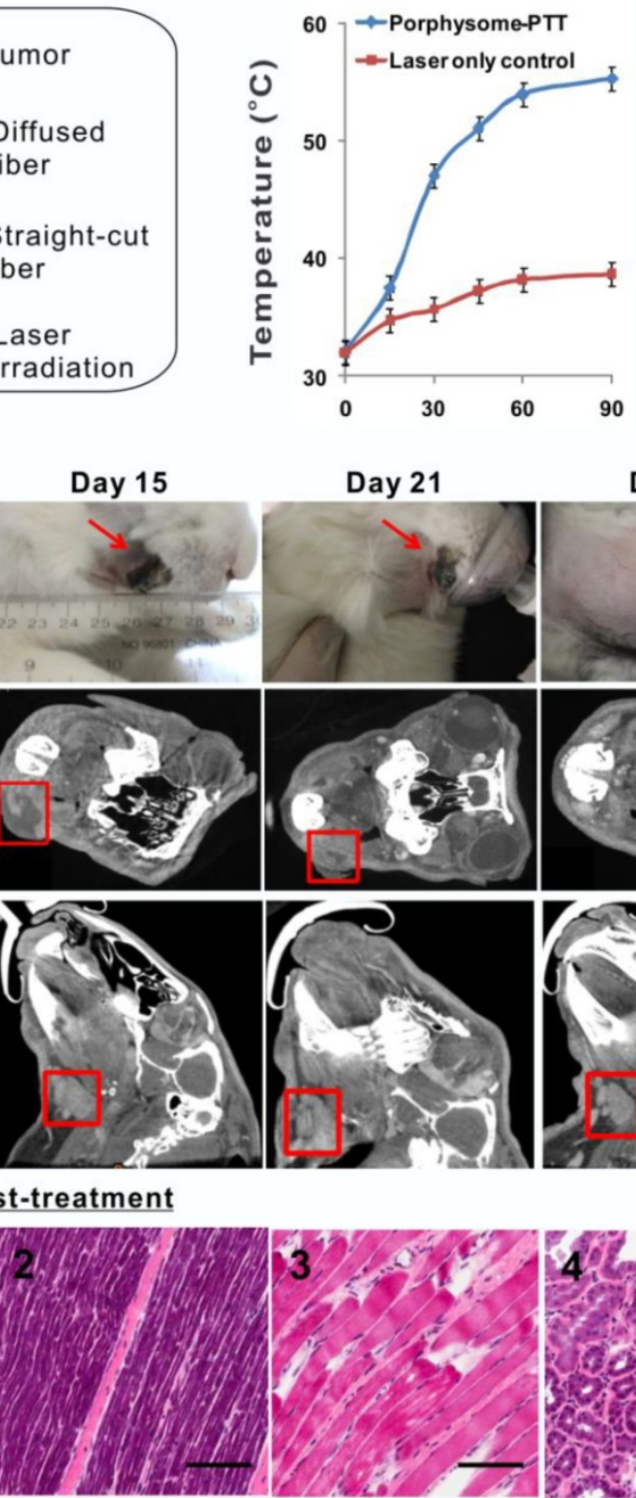

2
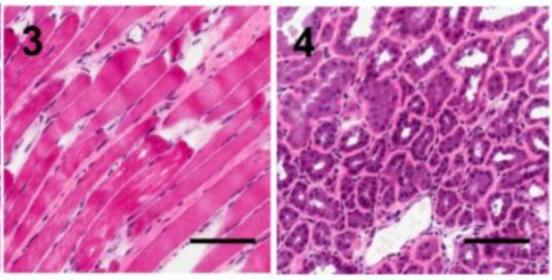

3

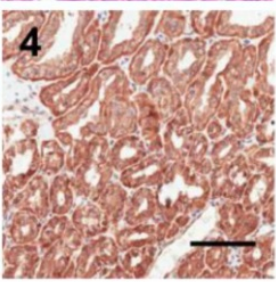

Figure 7. Porphysomes enabled imaging-guided PTT in treating rabbit oral tumor. A, scheme of experiment flow; B, temperature increase of tumor during PTT irradiation ( $\mathrm{n}=3$ for each group to calculate mean and standard deviation); $\mathbf{C}$. After porphysome-PTT, the size of tumor and lymph node was monitored by both bright-field white light imaging and CT scanning; $\mathbf{D}$, histological analysis of surgical bed at day 35 post-treatment by both $\mathrm{H} \& \mathrm{E}$ and pancytokeratin staining slides (scale bar: $50 \mu \mathrm{m}$.)

\section{Porphysomes-localized photothermal therapy induced complete ablation of tumor}


temperature of tumors from $32.00 \pm 0.17{ }^{\circ} \mathrm{C}$ to $55.30 \pm$ $0.22{ }^{\circ} \mathrm{C}(\mathrm{n}=3)$, while laser irradiation alone (without porphysomes administration) only caused the tumor temperature to increase from $31.90 \pm 0.21^{\circ} \mathrm{C}$ to $38.70 \pm$ $0.76{ }^{\circ} \mathrm{C}(\mathrm{n}=3)$, indicating that porphysomes are capable of targeted, potent PTT without resulting in undesired ablation of surrounding healthy tissues (Figure 7B).

The size of the tumors and metastatic cervical lymph nodes were monitored by palpation and volumetric measurements of 3D reconstructed micro-CT imaging prior to PTT and twice weekly after PTT (Figure 7C). Noticeably, a scar was observed on the tumor surface following treatment, which diminished gradually over a period of 35 days post-treatment (Figure 7C, i). A slight size increase of tumor was observed within 3 days after PTT treatment, which was likely due to the potent thermal ablation-induced inflammation and edema (Figure 7C, ii). The size of the tumors subsequently decreased gradually except for that of rabbit 3 of which the tumor size eventually began to decrease from day 15 onward (Figure S2A). Ultimately, all rabbits were tumor-free at day 35 . It was also observed that the volume change of the cervical lymph nodes exhibited similar trend to that of tumors (Figure 7C, iii). Eventually, the enlarged cervical lymph nodes size returned to normal once the primary tumor had completely diminished by day 35 . In contrast, the tumor and lymph node volumes in three control groups (a. no treatment, b. porphysome alone without PTT, c. PTT treatment alone without porphysomes) displayed continually increasing and

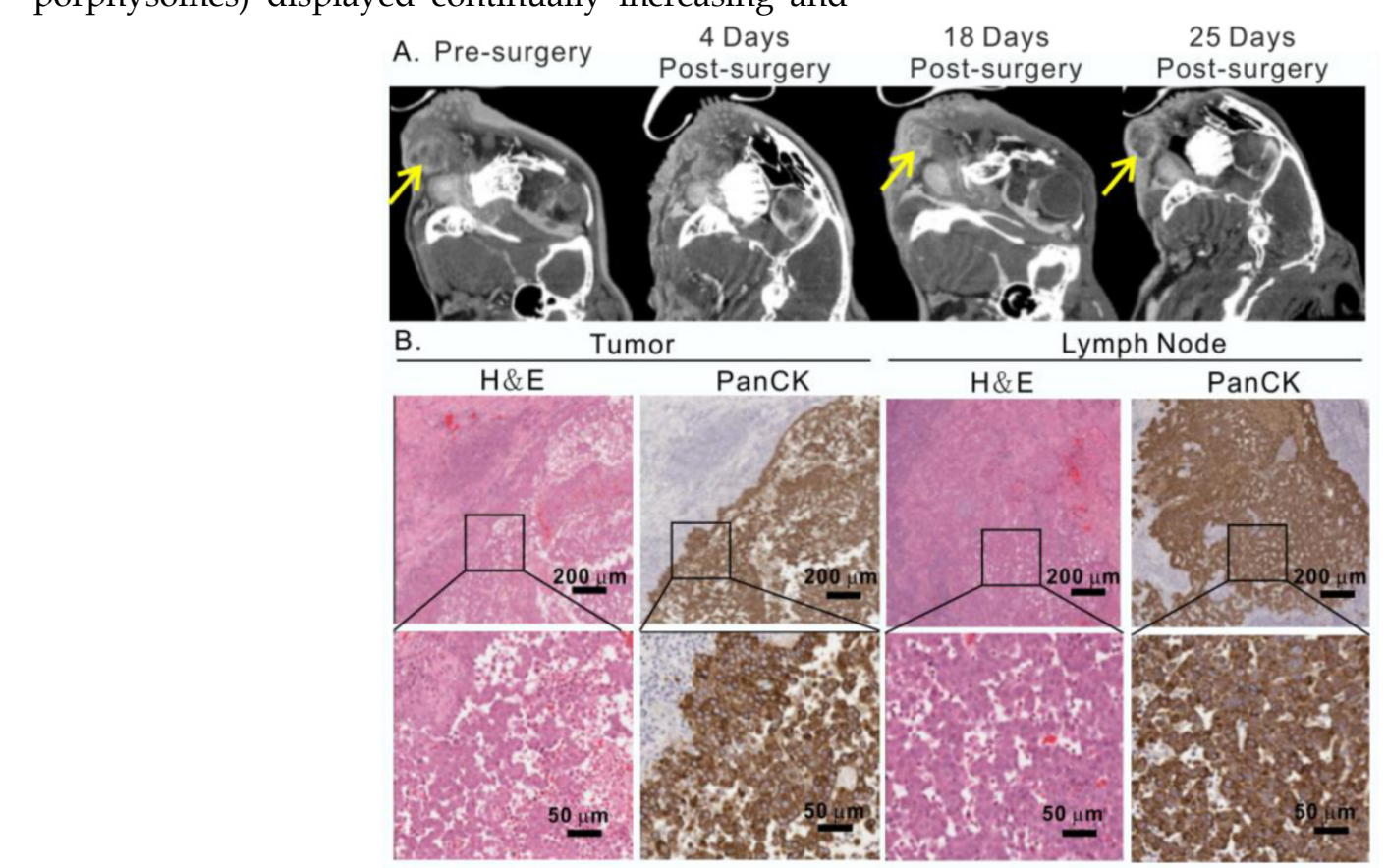

Figure 8. Conventional surgical treatment of rabbit oral tumor. A, After radical surgical resection of the tumor and cervical lymph nodes, the head and neck area was monitored by CT scan, showing the representative CT scan of tumor before and after surgery; $\mathbf{B}$, recurrence of tumor and metastasis in lymph node confirmed by

$\mathrm{H} \& \mathrm{E}$ and pancytokeratin staining analysis at 25 day post-surgery. reached the defined endpoint $\left(10000 \mathrm{~mm}^{3}\right)$ within in a week (Figure S2B). Complete tumor ablation of the porphysome-PTT group was confirmed by pathological analysis of the tissues resected from the original tumor area at day 35 post treatment, which did not exhibit any signs of tumor cell morphology (Figure 7D). As expected, the healthy salivary gland, due to its epithelial nature, displayed positive PanCK staining. However, the corresponding H\&E staining did not exhibit abnormal histology, indicating the absence of malignant cells. Altogether these findings suggest that porphysome-enhanced PTT enabled specific and effective tumor ablation, ultimately resulting in normalization of histological phenotype of the cheeks with previous tumor growth.

Moreover, the treatment outcome of porphysome-PTT was compared with a group of tumor-bearing rabbits $(n=3)$ that underwent conventional radical surgical resection of the tumor and cervical lymph nodes. However, surveillance CT scans and post-operative clinical examination revealed tumor local recurrences 18 days after surgery (Figure 8A, Figure S2C). The histology analysis from H\&E and positive PanCK staining further confirmed the recurrence of cancer cells in both primary tumor and lymph node (Figure 8B). These findings not only confirmed the aggressive behavior of VX2 tumors, but also reassured the effectiveness of porphysome-facilitated PTT for complete tumor ablation and successful management of buccal tumors in our HNSCC rabbit model. 


\section{a. Blood test parameters}
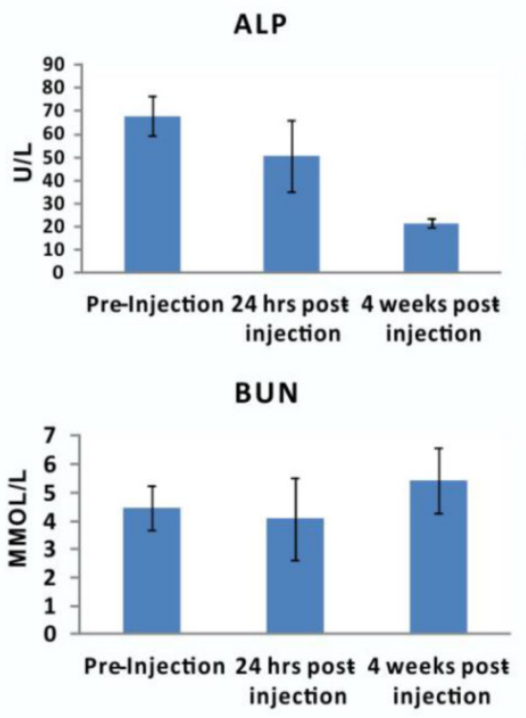

RBC

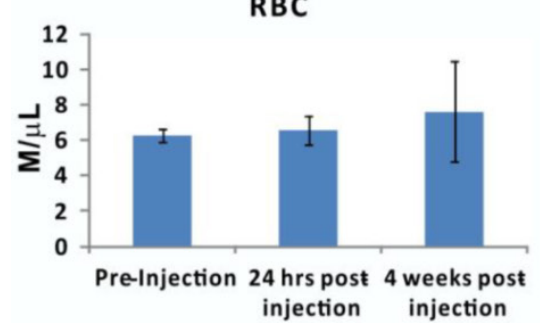

ALT

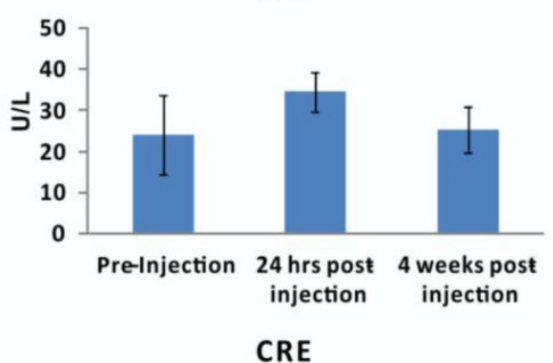

CRE

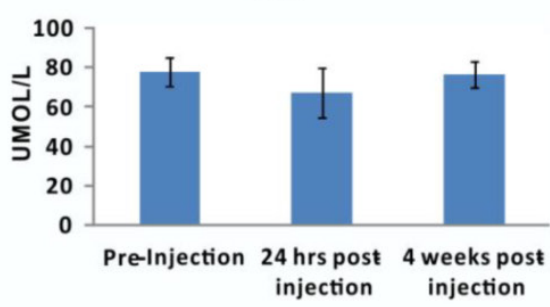

$\mathrm{Hb}$

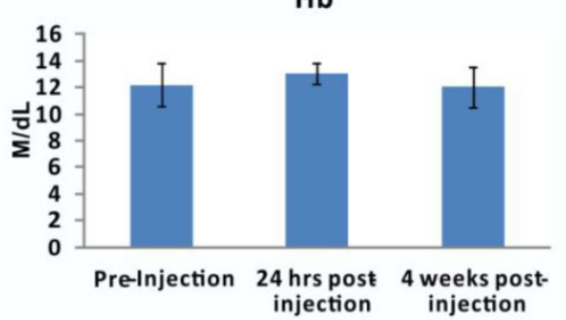

AMY
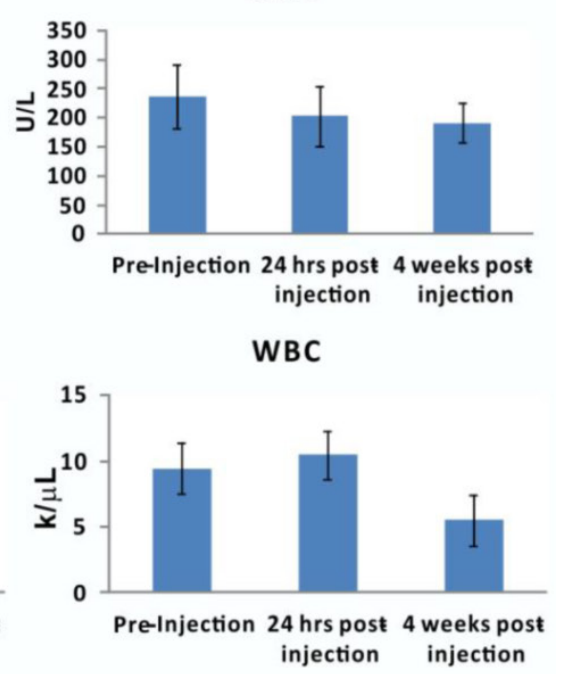

HCT

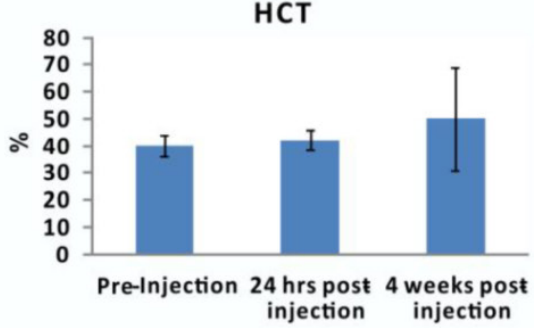

b. H\&E stained section of organs at 4 weeks post-injection

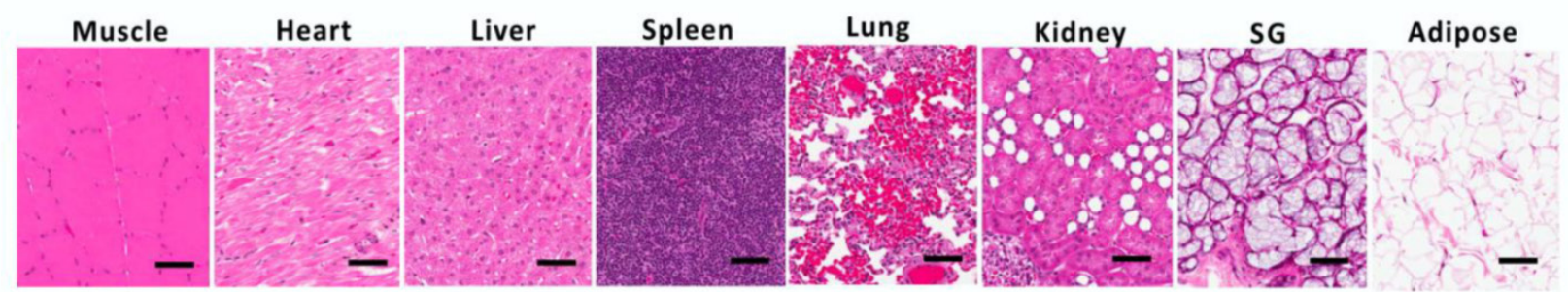

Figure 9. Evaluation of porphysome biocompatibility and toxicity in rabbits. A, Blood assay of rabbits before porphysome-injection, $24 \mathrm{~h}$ post-injection, and 4 weeks post-porphysome-PTT $(n=3)$; b) B, Representative H\&E staining analysis of organs of rabbit after 4 weeks porphysome-PTT (scale bar: $50 \mu \mathrm{m}$ ).

\section{Biocompatibility of porphysomes}

The biological safety and compatibility of porphysomes in systemic administration and PTT were assessed by blood tests on rabbits. The blood samples were collected for analysis prior to porphysomes injection, $24 \mathrm{~h}$ post-injection, and four weeks post-porphysome-PTT. Compared to pre-injection, 24 $\mathrm{h}$ post-porphysome administration and 4 weeks post-PTT did not exhibit significant changes with respect to level of alanine transaminase (ALT), amylase (AMY), blood urea nitrogen (BUN), creatinine (CRE), white blood cell (WBC), red blood cell (RBC), hemoglobin $(\mathrm{Hb})$ and hematocrit $(\mathrm{HCT})$, although alkaline phosphatase (ALP) levels decreased 4 weeks post-PTT but still within a normal range (Figure 9A). At 4 weeks post-porphysome-PTT, none of the organs of interest exhibited abnormal structural changes (including heart, liver, spleen, lung, kidney, salivary gland), and their histology analysis did not show any morphological abnormalities (Figure 9B). Thus, porphysomes-PTT appears to be biocompatible with our rabbit model.

\section{Discussion}

Effective management of HNSCC requires reliable means of tumor detection and targeted treatment modalities. Considering the critical anatomical structures surrounding head and neck tumors (i.e. cranial nerves, sensory organs, major blood vessels), it is crucial for treatment approaches to be specific, effective, and minimally invasive. Despite the large num- 
ber of nanoparticles that have been investigated for therapeutic and/or imaging purposes, very few are capable of fulfilling both imaging and therapeutic functionality while yielding effective outcomes without conferring adverse side effects. As a result, this work was motivated by the goal of assessing the efficacy of porphysomes to aid HNSCC management by enhancing head and neck tumor detection by both photoacoustic and fluorescence imaging and improving targeted treatment by localized PTT.

First of all, based on investigations on orthotopic rabbit and hamster HNSCC models, we have demonstrated that porphysomes can be used not only to delineate primary tumors, but also to localize metastatic lymph nodes through real-time intraoperative fluorescence and photoacoustic imaging. Porphysomes accumulated in tumor cells preferentially, as shown in the biodistribution result and histology analysis, which consequently enabled tumor-specific imaging. Due to the unique nanostructure of porphysomes, their imaging properties are a function of the degree to which their structure remains intact. The fluorescence of porphysome nanoparticle is highly-silenced due to the extremely high density of porphyrin in the intact particle [10]. Upon passive accumulation of porphysomes in tumors, porphysomes dissociate gradually and locally in tumor interstitial space. Even a small amount of dissociated porphysomes result in strong fluorescence signals because of the high porphyrin payload of porphysome, while the remaining intact porphysome nanoparticles enable photoacoustic imaging and PTT. Therefore, the nanostructure and unique photonic and physiochemical properties make porphysome a viable candidate for multimodal imaging and targeted therapy [21]. The performance of porphysomes as an accurate optical tracer for image-guided surgery and therapy was also evaluated in this study. There is an optimized time point that fulfills the needs of both fluorescence imaging and PTT, and in this study; we observed that $24 \mathrm{~h}$ post-injection of porphysomes was the best time point for image-guided therapy of HNSCC. Compared to many other nanoparticles that have been proposed for fluorescence imaging [22, 23], porphysomes achieved high tumor-to-tissue ratio of fluorescence with relatively lower background signal at $24 \mathrm{~h}$ post-injection, showing not only the primary tumor but also metastatic lymph nodes around the head and neck region. Furthermore, the photoacoustic signals enabled by porphysomes not only allowed visualization of tumors but also have the potential to indicate the extent of intact nanoparticles available for potent PTT outcomes, allowing investigators to determine the optimal time point(s) for efficient PTT.

As nodal staging is critical for HNSCC man- agement and prognosis, the fluorescence and photoacoustic imaging enabled by porphysomes can be explored to guide dissection of metastatic lymph nodes to facilitate the current sentinel lymph node biopsy (SLNB) protocol, where technetium-99 based SLNBs experience limitations on the lack of real-time intraoperative visual information [24-26].

Porphysomes did show some non-specific uptake in small intestine, adrenal glands, liver, spleen and kidneys, like many other nanoparticles. For example, gold-based nanoshells display much higher non-specific uptake in liver and spleen than in tumor (approximately 9-16 fold higher) [15]. However, these organs are anatomically distant from the head and neck region, so it is highly unlikely that they are capable of interfering with imaging and/or photothermal therapy in the head and neck region. Since liver and kidney functions levels were not dramatically altered upon porphysome injection, we can infer that kidney and liver functions were unlikely to be affected by porphysome uptake.

Porphysomes did not cause significant adverse side effects in large animal species either. Similar to previous observations in mice [10], the decrease of alkaline phosphatase levels and the white blood cell counts were also observed in rabbits after porphysome administration, but both remained within the normal ranges, indicating that porphysomes were not immunogenic. However, multiple blood test parameters in rabbits exhibited differences from that observed in mice [10]. For example, alanine transferase levels in rabbits at four weeks post-porphysome injection were similar to their pre-injection baseline levels, indicating normal liver function, while this parameter showed huge variations in mice [10]. Red blood cell counts, hemoglobin, and hematocrit levels were also largely unaffected in rabbits when compared to that in mice. The differences in blood cell parameters between rabbits and mice suggest that physiological differences and location of tumor might play a critical role in response to porphysome administration. In addition, physical examination of organs (heart, liver, spleen, lung, kidney, slavery gland, adipose, etc.) during tissue resection did not show any macroscopic abnormalities, which was confirmed with post-mortem histology analysis, indicating that there were no adverse effects on these organs. Therefore, although there appears to have been some undesirable, non-specific uptake of porphysomes in reticuloendothelial organs, our rabbit study demonstrated that the organs' functions were likely to have not been affected by porphysomes, thus emphasizing biocompatibility of porphysomes. Future clinical investigations are warranted to assess the uptake of porphysomes by reticuloendothelial organs in hu- 
mans, and to reaffirm whether these changes are consequential.

Considering complete tumor ablation in rabbits, it is reasonable to predict that porphysomes have the potential to significantly enhance targeted PTT. Photoacoustic and fluorescence dual image-guided tumor-localized PTT represents a minimally invasive technique that may serve as an alternative/complementary to surgery and reduce comorbidities and recurrences rate while enhancing treatment efficacy. In addition to complete tumor ablation after localized PTT irradiation, the size of the adjacent enlarged lymph node(s) significantly decreased to normal size even without receiving direct laser irradiation. One possibility for this interesting finding is that the PTT-treated tumors no longer release metastatic tumor cells into lymph drainage, thus causing the size decrease of the lymph node(s) and ultimately approving no existence of malignant cells of lymph nodes by pathology analysis.

The final temperature of tumor (effective therapeutic temperature) upon PTT induction in this study was consistent with that used in previous investigations with porphysomes and other porphyrin-based nanostructures [10,27]. Using light at wavelength of $671 \mathrm{~nm}$, the transmission depth of laser is limited to 5 $\mathrm{mm}$ to $1 \mathrm{~cm}$. The tumors of rabbit and hamster model were both superficial, which enabled the laser to irradiate the entire cancer tissue. However, for the tumors located deep in the tissue or that are less accessible, an alternative laser delivery device will be required, such as an endoscopic fiber that not only detects fluorescence signal for image-guidance but also delivers laser for localized PTT.

Overall, porphysomes propose minimal barriers to clinical translation, such as: 1) robust scale-up following proven liposome technology, 2) extremely low toxicity in mice by high dose of systemic administration $(1000 \mathrm{mg} / \mathrm{kg})$ and 3) biodegradable nature in vivo. This study of dual image-guided tumor localized PTT in large animal models substantiate the clinical potential of porphysomes to treat HNSCC patient for effective eradication of tumors without damage to adjacent critical structures. The next step must include the evaluation of porphysomes in clinical studies for further validation.

In summary, we demonstrated that porphysome not only enabled photoacoustic and fluorescence imaging of oral cavity carcinomas in rabbit and hamster models, but also allowed for tumor-localized PTT. Here we proposed that PTT could be precisely guided by both fluorescence imaging (control of laser placement and tumor delineation) and photoacoustic imaging (tumor margin delineation and assessment of effective PTT doses) for effective therapeutic efficacy.
This versatile and multifunctional nanoparticle possesses immense potential to be integrated into clinical care not only for HNSCC patients, but also for carcinomas originating from the breast, ovaries, lung, prostate, etc.

\section{Materials and Methods}

\section{Formation of porphysomes}

Porphysomes were synthesized using a previously reported protocol [28]. The lipid film of porphysomes consists of $55 \mathrm{~mol} \%$ of porphyrin-lipid (pyropheophorbide-lipid), $40 \mathrm{~mol} \%$ of cholesterol (Avanti Polar Lipids, Alabaster, AL) and $5 \mathrm{~mol} \%$ of distearoyl-sn-glycero-3-phosphoethanolamine-Nmethoxy(polyetheneglycol) (PEG2000-DSPE, Avanti Polar Lipids). Lipid films were dried under a gentle stream of nitrogen gas and additional $1 \mathrm{~h}$ vacuuming. To prepare the particles, each lipid film was rehydrated with PBS buffer (150 mM, pH 7.5) at the concentration of $10 \mathrm{mg} / \mathrm{mL}$, and extruded through a polycarbonate membrane (pore size $=100 \mathrm{~nm}$ ) for 10 times. Final size of the particles was measured by dynamic light scattering (ZS90 Nanosizer, Malvern Instruments) and the porphyrin concentration was determined by UV/Vis spectrometer (Varian Inc., Palo Alto, CA). Porphysomes were kept sterile and stored at $4{ }^{\circ} \mathrm{C}$ prior to use.

\section{Porphysomes in serum imaging study}

Porphysomes were incubated in $90 \mathrm{vol} \%$ fetal bovine serum (FBS) at $37^{\circ} \mathrm{C}$ at a concentration of 100 $\mu \mathrm{M}$ (pyro concentration) for $0,6,24$ or $48 \mathrm{~h}$. Samples were transferred to polyethylene tubing (PE20, Intramedic) for fluorescence and photoacoustic imaging. Fluorescence images were obtained using a whole-body small animal imager (Cri Maestro: Caliper Life Science Inc.) using blue-light excitation (435-480 nm), $515 \mathrm{~nm}$ long pass detection and $1000 \mathrm{~ms}$ exposure time. Fluorescence images were spectrally unmixed and overlayed on grey scale images of the tubes. For fold unquenching analysis, region of interests were drawn across each sample, and a ratio of the average signal counts from the sample incubated at time $0 \mathrm{~h}$ to each incubation time was compared.

Photoacoustic imaging was conducted using a commercial photoacoustic imaging system (Vevo ${ }^{\circledR}$ LAZR: FUJIFILM VisualSonics, Inc.), equipped with a $21 \mathrm{MHz}$ transducer in photoacoustic mode. 3D images were obtained at $680 \mathrm{~nm}$ with a $35 \mathrm{~dB}$ gain setting. For quantitative analysis, region of interests were outlined for each tube and the photoacoustic values were compared. Each sample was measured in triplicate and values were expressed as a mean \pm standard deviation.

Fluorescence spectra of each sample were ob- 
tained using a Fluoromax ${ }^{\circledR}$ fluorometer (Horiba Jobin Yvon) with an excitation wavelength of $410 \mathrm{~nm}$ and a $5 \mathrm{~nm}$ slit width. Fluorescence emission was collected from $600-800 \mathrm{~nm}$. Fluorescence spectra was also collected for the $48 \mathrm{~h}$ incubation sample with and without the addition of $0.5 \mathrm{vol} \%$ Triton-X100 in order to determine the percentage of fluorescence unquenching that had occurred by $48 \mathrm{~h}$. Porphysome samples were diluted in PBS to a concentration of $1 \mu \mathrm{M}$ pyro and measured using $410 \mathrm{~nm}$ excitation, $600-800 \mathrm{~nm}$ emission and $2 \mathrm{~nm}$ slit widths with and without the addition of detergent. Fluorescence unqueching was determined by dividing the sum of the fluorescence intensity from $650 \mathrm{~nm}$ to $750 \mathrm{~nm}$ with and without detergent.

\section{Tumor induction in hamsters and rabbits}

All animals received humane care in accordance with the policies formulated by the University Health Network (UHN) Animal Care Committee, the Animal for Research Act of the Province of Ontario, and the Canadian Council on Animal Care. All animal studies have been approved by the UHN Animal Care Committee protocols. Hamster orthotopic head and neck cancer models and a rabbit VX2 Head and neck tumor model were used throughout the study.

For hamster studies, 6-8 weeks old male Syrian hamsters (Harlan, Indianapolis, USA) were used as a model to mimic the clinical manifestations of oral carcinoma in humans. To induce tumor growth, $0.5 \%$ DMBA (7,12-dimethylbenz(a)anthracene) in DMSO was applied with a non-absorbent material to both cheeks while the animals were anesthetized with isofluorane. Prior to the application, KimWipes were packed in the oral cavity to minimize spilling down the throat, and were removed after the application was completed. This procedure was repeated three times a week, for a period of 16-20 weeks. Generally, visible tumors were observed after 10 weeks of DMBA application, and may reach up to $5-10 \mathrm{~mm}$ in size.

Male New Zealand white rabbits (Charles River, Wilmington, MA, USA) weighing from 2.0 to $3.5 \mathrm{~kg}$ were injected with $300 \mu \mathrm{L}$ of VX-2 tumor cell suspension $\left(5 \times 10^{6} / \mathrm{mL}\right)$ to induce tumor into the buccinator muscle. Tumors formed at the site of VX-2 cell injection and all rabbits presented with at least one cervical lymph node metastasis at two weeks post inoculation.

\section{Fluorescence imaging of tumors in hamsters and rabbits}

An in-house fluorescence imaging endoscopy system ( $650 \pm 20 \mathrm{~nm}$ excitation, $700 \pm 25 \mathrm{~nm}$ emission) was used to investigate the fluorescence imaging capacity of porphysomes. Prior to porphysome injection, bright field images and auto-fluorescence images of tumors were detected at the exposure time of 1500 $\mathrm{ms}$ for both rabbits and hamsters. $20 \mathrm{mg} / \mathrm{kg}$ of porphysomes (total lipid amount) was systemically administrated into the cephalic vein of hamsters and veins in the ears of rabbits. At $24 \mathrm{~h}$ post-intravenous injection, the tumors, at the same position as in the pre-injection images, were imaged with the same settings to obtain both the bright field and fluorescence images. Guided by the fluorescence of porphysome detected by endoscopy system, each tumor was dissected for ex vivo studies, and the bright field and fluorescence images were captured to ensure no tumor nodules were left on the surgical bed of animal cheek. In rabbit models, cervical lymph nodes were first located by physical palpation, and subsequently imaged by the fluorescence system before and $24 \mathrm{~h}$ after injection of porphysome, at which point, the fluorescent lymph nodes were dissected. Dissected tissues were imaged by the Maestro ${ }^{\circledR}$ system (with a 575-605 $\mathrm{nm}$ excitation filter and a $645 \mathrm{~nm}$ long-pass emission filter, with the exposure time of $500 \mathrm{~ms}$ ), and subsequently frozen for detailed histology analysis.

\section{Histology analysis}

To further investigate the porphysome accumulation at cellular level from the fluorescence-guided tissues dissection, frozen sections were used for histology analysis. Each tissue was sectioned to three adjacent slides of $5 \mu \mathrm{m}$ thickness each for three different staining groups (1. DAPI + Pyro 2. H\&E 3. Pancytokeratin (PanCK)). One slide was imaged by fluorescence microscopy using $633 \mathrm{~nm}$ laser channel for porphyrin and $408 \mathrm{~nm}$ laser channel for nuclei staining (DAPI), one slide was stained with PanCK to identify tumor cells, and the third slide was stained with H\&E for morphology analysis of the area of interest. Re-registration of the three images was used to evaluate the microscopic distribution of porphysomes in the resected tissue

\section{Porphysome biodistribution in hamster model}

The biodistribution of porphysomes in rabbit head and neck cancer was reported in a separate paper. Here we mainly assessed the in vivo biodistribution and pharmacokinetics of porphysomes in hamsters. $20 \mathrm{mg} / \mathrm{kg}$ of porphysomes (total lipid amount) was systemically administrated into the cephalic vain of hamsters. At $24 \mathrm{~h}$ post- injection of porphysomes, hamsters were euthanized and the organs of interest were dissected for further ex vivo analysis, including tumors, healthy mucosa, heart, kidney, spleen, small intestine, large intestine, and hind limb muscle. We used both a wide field-of-view hyperspectral fluorescence imager (Maestro EX 2.10, Cambridge Research \& Instrumentation, Inc., Waltham, MA) for 
macroscopic passive targeting of porphysomes and a laser confocal microscope for microscopic distribution of porphysomes in tumor. The animals without porphysome injection were used as controls. First, porphysome accumulation in head and neck tumor was semi-quantitatively detected and compared to other organs of interest by ex vivo fluorescence using Maestro® system (with a 575-605 nm excitation filter and a $645 \mathrm{~nm}$ long-pass emission filter, with the exposure time of $500 \mathrm{~ms}$ ), because Maestro focused more on the fluorescence of organ surface. Furthermore, biodistribution analyses were conducted to quantitatively measure porphysome accumulation in tumor and other organs. Here, organs of interest were weighed and homogenized in PBS. The porphyrin fluorescence in the supernatant was measured by fluoro-spectrometer, and \% of injected dose per gram (\% ID/g) of tissue was calculated based on a standard curve to calibrate pyro-lipid concentration. A total of 3 hamsters were used to calculate the average accumulation of porphysomes in various organs.

\section{Photoacoustic imaging of tumors in rabbits and hamsters}

Ultrasound and photoacoustic images were acquired with a commercial photoacoustic imaging system (Vevo® LAZR: FUJIFILM VisualSonics, Inc.), operating with a $21 \mathrm{MHz}$ transducer in both ultrasound and photoacoustic modes at $15 \mathrm{~dB}$ and $40 \mathrm{~dB}$ gains, respectively. For the rabbit head and neck cancer model, rabbits were injected with porphysomes and then sacrificed $24 \mathrm{~h}$ after injection. They were then decapitated and halved in the sagittal plane. The ex vivo tumor and cheek with no tumor were imaged at $710 \mathrm{~nm}$ transorally. For the hamster oral cancer model, animals were imaged in vivo transorally before and $24 \mathrm{~h}$ after porphysome injection using a wavelength of $680 \mathrm{~nm}$.

\section{Photothermal ablation tumors in hamsters and rabbits}

Hamster tumors were exposed to PTT when their size reached $4-5 \mathrm{~mm}$ in diameter, measured manually by ruler. $24 \mathrm{~h}$ post- Porphysomes injection (20 mg/ $\mathrm{kg}$ of total lipid), fluorescence imaging was used to aid in the identification of tumor in order to guide the placement of the PTT laser. PTT irradiation was conducted using a straight-cut fiber $(671 \mathrm{~nm})$. The fiber height was adjusted so that the irradiation area was $8 \mathrm{~mm}$ in diameter to cover the entire tumor area. The output was set to $700 \mathrm{~mW}$ and the laser was on for 100 seconds. The total laser energy was $100 \mathrm{~J} / \mathrm{cm}^{2}$. Temperature of tumor was monitored by a near-infrared thermal camera once every 10 seconds as a real-time evaluation of PTT efficacy. Tumors of hamsters without porphysome injection were used as controls. 3 hamsters were used for each group.

Rabbits were injected with $20 \mathrm{mg} / \mathrm{kg}$ porphysomes via ear vein when the tumors reached a diameter of $1 \mathrm{~cm}$, monitored by both CT images and ruler. $24 \mathrm{~h}$ after the injection, animals were anesthetized with $2 \%$ isofluorane and two PTT irradiations were performed: For the first treatment, a diffused-fiber was inserted into tumor ( $1 \mathrm{~W}, 9 \mathrm{~mm}$ length, $90 \mathrm{~s})$ to irradiate the bottom half of tumor. When irradiation was completed, the diffused-laser was taken out and a straight-cut fiber was used to irradiate tumor from the surface, as the second treatment. The area of irradiation was adjusted to $1.5 \mathrm{~cm}$ diameter to cover the entire tumor area from the outside $(1.5 \mathrm{~W}, 90$ seconds). In total, $200 \mathrm{~J}$ PTT laser energy was delivered to ablate the head and neck tumor in rabbits. Three control groups were also included, (a) non-treatment control; (b) porphysome-alone group $(20 \mathrm{mg} / \mathrm{kg})$, and (c), laser alone control group.

\section{Post-treatment evaluation of porphy- some-enhanced PTT}

Since there were multiple tumors on the hamsters' cheeks, the hamster tumor model was deemed to be too aggressive for survival study, and we only used it to investigate the acute PTT response by histology analysis. We evaluated the long-term therapeutic efficacy of porphysome-PTT on rabbit model by survival study.

In the hamster model, animals were euthanized shortly after tumor was irradiated by PTT laser. Tumors were dissected and frozen in OCT gel using liquid nitrogen. Frozen tumor was sectioned to be $5 \mu \mathrm{m}$ and stained with H\&E. The acute response of porphysome-PTT was analyzed based on morphology properties of tumor cells.

In the rabbit model, tumor size was monitored by both CT imaging and manual measurement with ruler once every 3-4 days after treatment. The growth of tumor and lymph node of rabbits in each group were plotted individually, designating day 0 as the time when treatment was performed. Rabbits were not sacrificed until tumor reached the defined end point $(2 \mathrm{~cm}$ diameter). In the porphysome-enhanced PTT group, rabbits were sacrificed after 30 days, and the cheek that originally had tumor grown was dissected and put in formalin for histology analysis. $\mathrm{N}=3$ at least in each group was used to calculate the average and standard deviation.

\section{CT Imaging}

MicroCT system (Locus Ultra: GE Healthcare, U.K.) was used to scan and monitor the tumor size in rabbit after treatment. An image of each rabbit with 
the head and neck tumor was collected immediately following $5 \mathrm{~mL}$ injections of Omnipaque 350 (GE Healthcare, Mississauga ON). Volumes of interest were drawn and countered on the CT images using Inveon Research Workplace (Siemens, Munich, Germany) to evaluate the tumor response to treatment.

\section{Acute and long-term toxicity of porphysomes}

To examine the toxicity of porphysomes, rabbit blood was collected from the ear vein for multiple blood tests at three time points: before the injection, 24 $\mathrm{h}$ post-injection and 4 weeks post-injection (porphysome dose at $20 \mathrm{mg} / \mathrm{kg}$ ). ALP, ALT, AMY, BUN, CRE, $\mathrm{WBC}, \mathrm{RBC}, \mathrm{Hb}$ and HCT were tested and $\mathrm{n}=3$ was used for averaged values and standard deviation. Additionally, for evaluation of long-term toxicity, a group of rabbits $(n=3)$ received porphysome injection and were euthanized one month later. Organs of interest (liver, spleen, kidney, heart, lung, muscle, salivary gland, fat) were collected and fixed in formalin for H\&E staining, and morphology of each organ was compared to the organs from healthy rabbit without porphysome injection, as the evaluation of long-term toxicity.

\section{Statistic analysis}

All the values were presented as mean and standard deviation (SD), and the Student's t-test (two-tailed) was used to determine the significance of the difference between two groups of values, and $\mathrm{P}<0.05$ was considered significant.

\section{Supplementary Material}

Figures S1-S2. http:/ / www.thno.org/v05p1428s1.pdf

\section{Acknowledgements}

The authors are thankful to the Princess Margaret Cancer Foundation, the Canadian Institutes of Health Research, the Ontario Institute for Cancer Research, the Natural Sciences and Engineering Research Council of Canada, the Canada Foundation for Innovation, Terry Fox New Frontiers Program Project Grant, and the Joey and Toby Tanenbaum/Brazilian Ball Chair in Prostate Cancer Research for funding this work. In addition, the authors would like to thank Sandy Lafrance, Maria Bisa, Dr. Badru Maloo, Dr. Janet Sunohara-Neilson and Alyssa Goldstein for their animal care support, Deborah Scollard for assistance with CT imaging, and Tahsin Khan and Cindy Hsin for imaging analysis.

\section{Competing Interests}

The authors have declared that no competing interest exists.

\section{References}

1. Pulte D, Brenner H. Changes in survival in head and neck cancers in the late 20th and early 21st century: a period analysis. Oncologist. 2010; 15: 994-1001.

2. The Department of Veterans Affairs Laryngeal Cancer Study Group. Induction chemotherapy plus radiation compared with surgery plus radiation in patients with advanced laryngeal cancer. The New England journal of medicine. 1991; 324: 1685-90.

3. Forastiere AA, Goepfert H, Maor M, Pajak TF, Weber R, Morrison W, et al. Concurrent chemotherapy and radiotherapy for organ preservation in advanced laryngeal cancer. The New England journal of medicine. 2003; 349: 2091-8.

4. Parsons JT, Mendenhall WM, Stringer SP, Amdur RJ, Hinerman RW, Villaret $\mathrm{DB}$, et al. Squamous cell carcinoma of the oropharynx: surgery, radiation therapy, or both. Cancer. 2002; 94: 2967-80.

5. Liederbach E, Lewis CM, Yao K, Brockstein BE, Wang CH, Lutfi W, et al. A Contemporary Analysis of Surgical Trends in the Treatment of Squamous Cell Carcinoma of the Oropharynx from 1998 to 2012: A Report from the National Cancer Database. Annals of surgical oncology. 2015.

6. O'Neill CB, Baxi SS, Atoria CL, O'Neill JP, Henman MC, Sherman EJ, et al. Treatment-related toxicities in older adults with head and neck cancer: A population-based analysis. Cancer. 2015; 121: 2083-9.

7. Wang Y, Black KC, Luehmann H, Li W, Zhang Y, Cai X, et al. Comparison study of gold nanohexapods, nanorods, and nanocages for photothermal cancer treatment. ACS nano. 2013; 7: 2068-77.

8. Jori G, Spikes JD. Photothermal sensitizers: possible use in tumor therapy. J Photochem Photobiol B. 1990; 6: 93-101.

9. Goldberg SN, Gazelle GS, Mueller PR. Thermal ablation therapy for focal malignancy: a unified approach to underlying principles, techniques, and diagnostic imaging guidance. AJR Am J Roentgenol. 2000; 174: 323-31.

10. Lovell JF, Jin CS, Huynh E, Jin H, Kim C, Rubinstein JL, et al. Porphysome nanovesicles generated by porphyrin bilayers for use as multimodal biophotonic contrast agents. Nature materials. 2011; 10: 324-32.

11. Jin CS, Lovell JF, Chen J, Zheng G. Ablation of hypoxic tumors with dose-equivalent photothermal, but not photodynamic, therapy using a nanostructured porphyrin assembly. ACS nano. 2013; 7: 2541-50.

12. Liu TW, Macdonald TD, Jin CS, Gold JM, Bristow RG, Wilson BC, et al. Inherently Multimodal Nanoparticle-Driven Tracking and Real-Time Delineation of Orthotopic Prostate Tumors and Micrometastases. ACS nano. 2013; 7: 4221-32.

13. Yang K, Zhang S, Zhang G, Sun X, Lee ST, Liu Z. Graphene in mice: ultrahigh in vivo tumor uptake and efficient photothermal therapy. Nano Lett. 2010; 10: 3318-23.

14. Lal S, Clare SE, Halas NJ. Nanoshell-enabled photothermal cancer therapy: impending clinical impact. Acc Chem Res. 2008; 41: 1842-51.

15. Melancon MP, Lu W, Zhong M, Zhou M, Liang G, Elliott AM, et al. Targeted multifunctional gold-based nanoshells for magnetic resonance-guided laser ablation of head and neck cancer. Biomaterials. 2011; 32: 7600-8.

16. von Maltzahn G, Park JH, Agrawal A, Bandaru NK, Das SK, Sailor MJ, et al. Computationally guided photothermal tumor therapy using long-circulating gold nanorod antennas. Cancer Res. 2009; 69: 3892-900.

17. Ren F, Bhana S, Norman DD, Johnson J, Xu L, Baker DL, et al. Gold nanorods carrying paclitaxel for photothermal-chemotherapy of cancer. Bioconjug Chem. 2013; 24: 376-86.

18. Chen J, Glaus C, Laforest R, Zhang Q, Yang M, Gidding M, et al. Gold nanocages as photothermal transducers for cancer treatment. Small. 2010; 6: 811-7.

19. Cornella FA, Saper CD, Christensen RE, Park NH. Effect of DMBA on oral cancer development in hamsters with latent HSV-1 infections in trigeminal ganglia. Oral surgery, oral medicine, and oral pathology. 1989; 67: 167-71.

20. Chen YK, Lin LM . DMBA-induced hamster buccal pouch carcinoma and VX2-induced rabbit cancer as a model for human oral carcinogenesis. Expert review of anticancer therapy. 2010; 10: 1485-96.

21. Huynh E, Zheng G. Engineering multifunctional nanoparticles: all-in-one versus one-for-all. Wiley Interdiscip Rev Nanomed Nanobiotechnol. 2013; 5: 250-65.

22. Yang Z, Zheng S, Harrison WJ, Harder J, Wen X, Gelovani JG, et al. Long-circulating near-infrared fluorescence core-cross-linked polymeric micelles: synthesis, characterization, and dual nuclear/optical imaging. Biomacromolecules. 2007; 8: 3422-8.

23. Yang H, Mao H, Wan Z, Zhu A, Guo M, Li Y, et al Micelles assembled with carbocyanine dyes for theranostic near-infrared fluorescent cancer imaging and photothermal therapy. Biomaterials. 2013; 34: 9124-33.

24. Burcia V, Costes V, Faillie JL, Gardiner Q de Verbizier D, Cartier C, et al. Neck restaging with sentinel node biopsy in T1-T2N0 oral and oropharyngeal cancer: Why and how? Otolaryngology--head and neck surgery : official journal of American Academy of Otolaryngology-Head and Neck Surgery. 2010; 142: 592-7 e1.

25. de Rosa N, Lyman GH, Silbermins D, Valsecchi ME, Pruitt SK, Tyler DM, et al. Sentinel node biopsy for head and neck melanoma: a systematic review. Otolaryngology--head and neck surgery : official journal of American Academy of Otolaryngology-Head and Neck Surgery. 2011; 145: 375-82.

26. Koch WM, Choti MA, Civelek AC, Eisele DW, Saunders JR. Gamma probe-directed biopsy of the sentinel node in oral squamous cell carcinoma. Archives of otolaryngology--head \& neck surgery. 1998; 124: 455-9. 
27. Li Y, Lin TY, Luo $Y$, Liu Q, Xiao W, Guo W, et al. A smart and versatile theranostic nanomedicine platform based on nanoporphyrin. Nat Commun. 2014; 5: 4712.

28. Jin CS, Lovell JF, Zheng G. One minute, sub-one-watt photothermal tumor ablation using porphysomes, intrinsic multifunctional nanovesicles. J Vis Exp. 2013. 